\title{
Evaluación científico-técnica de la Ley General para el Control del Tabaco de México
}

\author{
Tonatiuh Barrientos-Gutiérrez, MD,MS,PhD. (I)
}

\begin{abstract}
Barrientos-Gutiérrez T. Evaluación científico-técnica de la Ley General para el Control del Tabaco de México. Salud Publica Mex 2010;52 supl 2:S277-S282.
\end{abstract}

\begin{abstract}
Resumen
La Ley General para el Control del Tabaco, aprobada en febrero de 2008, busca proteger a la población mexicana de los efectos nocivos del tabaco y asegurar el derecho de los no fumadores a vivir y convivir en espacios $100 \%$ libres de humo de tabaco. La Ley considera el desarrollo de espacios libres de humo, pero también posibilita la existencia de áreas interiores para fumar. El presente ensayo examina la Ley y su Reglamento, artículo por artículo, evaluando su capacidad para asegurar la protección a la salud de la población de los efectos del humo de tabaco ambiental. El análisis revela imprecisiones conceptuales y operativas importantes, pero principalmente, señala de manera puntual las razones por las que las áreas interiores para fumar representan un riesgo a la salud de la población. Se concluye que la Ley debe ser reformada, eliminando la provisión de áreas interiores para fumar, de cualquier estructura cubierta en las áreas para fumar al aire libre y definiendo la distancia mínima entre estas áreas y las áreas 100\% libres de humo de tabaco.
\end{abstract}

Palabras clave: humo de tabaco ambiental; políticas públicas; legislación; ambientes libres de humo de tabaco; México
Barrientos-Gutiérrez T.

Scientific and technical evaluation of Mexico's

General Law for Tobacco Control.

Salud Publica Mex 2010;52 suppl 2:S277-S282.

\section{Abstract}

The General Law for Tobacco Control, signed in February 2008, aims to protect the Mexican population against the negative effects associated with tobacco consumption and to guarantee the non-smoker's rights to live and relate in $100 \%$ smoke-free environments. The Law supports the development of smoke-free areas, but it also allows for indoor smoking areas. The present essay examines the Law and its Rule of Procedure, article by article, evaluating its capability to assure the protection of the population against the effects of environmental tobacco smoke. The analysis reveals conceptual and operative important imprecision, but mainly, discusses in detail the reasons why indoor smoking areas represent a health risk for the population. It concludes that the Law must be reformed, eliminating the provision of indoor smoking areas, any kind of roof in outdoor smoking areas and establising a minimum distance between these areas and the $100 \%$ smoke-free environments.

Key words: environmental tobacco smoke; public policy; legislation; smoke-free environments; Mexico
L a exposición al humo de tabaco ambiental (HTA) representa un problema global de salud pública. ${ }^{1-4}$ Existe consenso científico internacional sobre los efectos nocivos derivados de la exposición al HTA, que incluyen incrementos en el riesgo de enfermedad cardiovascular y cáncer de pulmón en adultos y síndrome de muerte súbita del lactante y diversas enfermedades respiratorias en niños. ${ }^{1,5,6}$
La complejidad química y peligrosidad del HTA ha obligado a la Organización Mundial de la Salud (OMS) a considerar cualquier nivel de exposición a este contaminante como potencialmente nocivo para la salud. ${ }^{5}$ Por ello, la prohibición al consumo de tabaco en espacios interiores es la única estrategia recomendada por la OMS para asegurar la prevención de los efectos nocivos del HTA. $^{2,5}$ Sin embargo, las compañías tabacaleras ${ }^{7,8}$ han

(I) Instituto Nacional de Salud Pública. México DF, México.

Fecha de recibido: 26 de marzo de 2010 • Fecha de aceptado: 13 de julio de 2010

Solicitud de sobretiros: Dr.Tonatiuh Barrientos. Departamento de Investigación sobre Tabaco. Centro de Investigación en Salud Poblacional. Instituto Nacional de Salud Pública. $7^{\text {a }}$ Cerrada de Fray Pedro de Gante 50, Col. Sección XVI. 14000 Del.Tlalpan, México DF.

Correo electrónico: tbarrientos@correo.insp.mx 
intentado desestimar la necesidad de la prohibición con el argumento de que la eliminación de los riesgos derivados del HTA puede lograrse mediante sistemas de ingeniería como separación de áreas, ventilación, extracción y purificación del aire. ${ }^{7}$ A pesar del rechazo de la OMS, esta postura ha encontrado eco en diversas legislaciones en el plano internacional. ${ }^{9}$

En México, la Ley General para el Control del Tabaco (LGCT) aprobada en febrero de 2008, define el marco legal vigente para el control del HTA. ${ }^{10} \mathrm{La}$ LGCT tiene como objetivo proteger la salud de la población de los efectos nocivos del tabaco y asegurar que los no fumadores puedan vivir y convivir en espacios libres de humo. Para ello, considera la creación de espacios 100\% libres de humo de tabaco, pero también permite la existencia de zonas exclusivas para fumar en interiores, que deben ajustarse a criterios reglamentarios que eviten la contaminación de las áreas adyacentes de no fumar.

El presente ensayo analiza desde un punto de vista científico y técnico las disposiciones reglamentarias de la Ley General para el Control del Tabaco (LGCT) en lo que respecta a las áreas interiores exclusivas para fumar y los espacios $100 \%$ libres de humo de tabaco, evaluando su capacidad para asegurar la protección de la salud de la población de los efectos nocivos del HTA.

Principios para la protección contra el HTA: Artículos 5, 26 y 27 de la LGCT

Los objetivos de la Ley General para el Control del Tabaco están descritos en el Artículo 5. Las fracciones correspondientes a la protección contra el HTA son:

Artículo 5. La presente Ley tiene las siguientes finalidades:

I. Proteger la salud de la población de los efectos nocivos del tabaco;

II. Proteger los derechos de los no fumadores a vivir y convivir en espacios $100 \%$ libres de humo de tabaco;

III. Establecer las bases para la protección contra el humo de tabaco;

$$
\text { (...) }
$$

Las medidas de protección contra el HTA están descritas en el Capítulo III de la LGCT, que define los principios rectores para la Protección contra la Exposición al Humo de Tabaco. El Artículo 26 instituye los espacios $100 \%$ libres de humo de tabaco al establecer que:

Artículo 26. Queda prohibido a cualquier persona consumir o tener encendido cualquier producto del tabaco en los espacios $100 \%$ libres de humo de tabaco, así como en las escuelas públicas y privadas de educación básica y media superior. (...)

La existencia de zonas exclusivas para fumar está sancionada en el Artículo 27 de la LGCT, que establece:

Artículo 27. En lugares con acceso al público, o en áreas interiores de trabajo, públicas o privadas, incluidas las universidades e instituciones de educación superior, podrán existir zonas exclusivamente para fumar, las cuales deberán de conformidad con las disposiciones reglamentarias:

I. Ubicarse en espacios al aire libre, $\mathrm{o}$

II. En espacios interiores aislados que dispongan de mecanismos que eviten el traslado de partículas hacia los espacios $100 \%$ libres de humo de tabaco y que no sea paso obligado para los no fumadores.

Así, la LGCT tiene como objetivo principal proteger a la población de los efectos nocivos del tabaco y asegurar que los no fumadores vivan en espacios 100\% libres de humo. Considerando estos objetivos, el principio fundamental para evaluar la utilidad de la LGCT es definir el estándar de protección que debe ofrecerse a la población para asegurar que no experimentará los efectos nocivos del HTA. Aunque para diversos contaminantes ambientales existen niveles máximos de exposición que no deben superarse a riesgo de dañar la salud, este criterio no puede aplicarse al HTA, ya que cualquier nivel de exposición es potencialmente nocivo. Por tanto, la protección efectiva de la población requiere forzosamente de la eliminación completa del contaminante. ${ }^{2}$ Bajo este principio, existen dos problemas fundamentales derivados de los artículos 26 y 27 de la LGCT.

El primer problema es la definición limitada de "espacio 100\% libre de humo". De acuerdo con el Artículo 6 Fracción X el espacio 100\% libre de humo de tabaco es:

Aquélla área física cerrada con acceso al público o todo lugar de trabajo interior o de transporte público, en los que por razones de orden público e interés social queda prohibido fumar, consumir o tener encendido cualquier producto de tabaco.

Esta definición prohíbe el consumo activo de tabaco y asegura que el área en cuestión será de "no fumar", sin embargo, no garantiza que el aire estará $100 \%$ libre de humo de tabaco. Bajo la definición proporcionada por la LGCT es posible que un espacio sea considerado 100\% libre de humo de tabaco a pesar de estar siendo conta- 
minado por fuentes externas, tales como el consumo de tabaco en los accesos o la transmisión de humo desde espacios adyacentes para fumar. El segundo problema se relaciona directamente y deriva de la existencia de áreas para fumar en interiores prevista en el Artículo 27. Hasta el momento, la evidencia científica disponible demuestra que no existe ningún mecanismo que evite el traslado de partículas desde las áreas de fumar, ${ }^{11}$ por lo que la instalación de éstas áreas necesariamente implicará la contaminación de los espacios adyacentes, lo que elimina la provisión de $100 \%$ libres de humo y vulnera el derecho a la salud de los no fumadores. Una explicación pormenorizada de esta limitación se proporciona a continuación, analizando los requerimientos sancionados en el Reglamento de la LGCT.

Protección contra el HTA: Artículos 60, 61 y 63 del Reglamento de la LGCT

Las especificaciones técnicas de la LGCT están descritas en el Reglamento de la LGCT (RLGCT) publicado el 31 de mayo de 2009. ${ }^{12}$ El Artículo 60 del RLGCT establece que para lograr la protección de la población del HTA:

Artículo 60. Las zonas exclusivamente para fumar deberán ubicarse al aire libre o en espacios interiores aislados y contar con las siguientes características:

I. Las que estén ubicadas al aire libre, deberán estar físicamente separadas e incomunicadas de los espacios $100 \%$ libres de humo, no ser paso obligado para las personas o encontrarse en los accesos o salidas de los inmuebles. En estos espacios no podrán estar menores de edad y deberá advertirse a las mujeres embarazadas de los riesgos que corre ella y el producto al entrar en esta zona, $\mathrm{y}$

II. En caso de tratarse de espacios interiores aislados, deberán cumplir con los requerimientos técnicos establecidos en la Ley, este Reglamento y demás disposiciones legales aplicables.

La Fracción I del Artículo 60 define las condiciones en las que deberán operar las áreas de fumar al aire libre para evitar la contaminación de los espacios 100\% libres de humo, incluyendo la separación física, el que no sea paso obligado y el que no se encuentre en accesos o salidas de los inmuebles. Esta fracción es necesaria porque la contaminación de las áreas interiores de no fumar desde áreas adyacentes de consumo al aire libre ha sido reportada anteriormente. ${ }^{13}$ Aunque la especificación reglamentaria a este respecto es integral, está limitada por una definición deficiente de "espacio al aire libre" y por la falta de precisión en cuanto a la distancia mínima que debe existir entre las áreas para fumar al aire libre y las áreas de paso obligado, las salidas y los accesos de los inmuebles.

El Reglamento define "espacio al aire libre" en su Artículo 2 Fracción IV como "... aquel que no tiene techo ni estál limitado entre más de una pared o muro, independientemente del material utilizado para su construcción y de que la estructura sea permanente o temporal. Para efectos de esta definición el concepto de techo no incluye sombrillas, palapas, tejabanes, techos abatibles o desmontables y lonas;". La permisividad de la Ley hacia estructuras como palapas, tejabanes o techos desmontables, que estructuralmente difieren poco de un techo de concreto, transforma el espacio al aire libre en un espacio semicerrado, eliminando el libre flujo de aire y permitiendo la concentración del humo de tabaco ambiental. Ante esta consideración, la OMS ha recomendado que no se permita el consumo de tabaco en espacios semicerrados. ${ }^{2}$ De esta manera, la definición de "espacio al aire libre" tendría que excluir la posibilidad de instalar cualquier estructura que pudiera funcionar como techo.

La falta de precisión en cuanto a la distancia mínima que debe existir entre las áreas de fumar y los espacios $100 \%$ libres de humo permite una interpretación subjetiva de la LGCT, vulnerando su aplicabilidad. Por ello, es necesario que la LGCT establezca con claridad la distancia mínima de las áreas de fumar a las áreas de no fumar y de paso obligado. Algunos ejemplos de legislaciones que han considerado distancias mínimas incluyen el Acta de Illinois Libre de Humo (4.5 metros) y la Ley de Hawaii Libre de Humo (6 metros). ${ }^{14,15}$

Las especificaciones reglamentarias para los espacios interiores aislados para fumar son presentadas en los Artículos 61 y 63 del RLGCT. Las fracciones I, II y IV del Artículo 61 establecen los requerimientos técnicos de dichos espacios:

Artículo 61. En los espacios interiores aislados se deberán cumplir los siguientes requisitos:

I. Encontrarse totalmente separado de piso a techo y de pared a pared de los espacios 100\% libres de humo de tabaco por todos sus lados;

II. Contar con una puerta de apertura y cierre automática con mecanismo de movimiento lateral, no abatibles; que permanecerá cerrada permanentemente y se abrirá únicamente durante el acceso o salida de esas zonas; (...)

IV. No representar un paso obligado para las personas.

Hasta esta fecha no existe evidencia científica que asegure que la separación de áreas provista en la Fracción I del Artículo 61 evite el traslado de HTA de 
las áreas de fumar a las de no fumar. Por el contrario, diversos estudios han mostrado que la separación de áreas es inútil para controlar la transmisión de humo de tabaco. ${ }^{2}$ Extensas investigaciones han sido llevadas a cabo en salas de fumar en aeropuertos, restaurantes e incluso entre vagones de tren exclusivos para fumar: en todos los casos ha existido transmisión de partículas y compuestos. ${ }^{16-18}$

La puerta de apertura lateral requerida por la Fracción II del Artículo 61 evita el mecanismo de bombeo de aire que se genera al abrir y cerrar una puerta de apertura frontal. Aunque la eliminación del bombeo reduce la transmisión, el uso de una puerta lateral no evita el paso de partículas de las zonas de fumar a las zonas de no fumar. Estudios experimentales muestran que el uso de puertas laterales reduce en un 77\% la transmisión de partículas que se observa con una puerta de apertura frontal. ${ }^{19}$ Sin embargo, esto implica que $23 \%$ aún se transmite a las áreas de no fumar. Finalmente, la Fracción IV del Artículo 61 presenta la misma dificultad que la Fracción I del Artículo 60, ya que no establece una distancia mínima de las áreas de fumar a las áreas de paso obligado para las personas.

Las características de los sistemas de ingeniería requeridas para las áreas exclusivas para fumar, están descritas en las Fracciones I, III y IV del Artículo 63:

Artículo 63. El espacio interior aislado deberá contar forzosamente con un sistema de ventilación y purificación que garantice lo siguiente:

I. Recambio de aire limpio, continuo y permanente, que corresponda al total del volumen interior por cada 20 minutos. No se podrán utilizar equipos de recirculación de aire; este mínimo suministro de aire puede transferir aire de otras zonas de no fumar del edificio o establecimiento, y debe mantenerse continuamente durante las horas de funcionamiento del local. Además este suministro mínimo de aire debe estar claramente consignado en el certificado de ocupación;

III. Aporte mínimo que asegure 30 litros de aire por segundo por persona dentro del espacio, sobre la base de un índice de aforo de 1 persona por cada 1.5 metros cuadrados;

IV. Mantener una presión negativa con el resto del establecimiento no inferior a 6 Pascales, la cual deberá registrarse automáticamente durante toda la jornada que el establecimiento permanezca abierto. (...)

El uso de sistemas de ventilación y purificación del aire propuesta en el Artículo 63 ha sido descartado como una opción eficaz para la eliminación de los riesgos a la salud derivados de la exposición al HTA. ${ }^{11}$ El HTA está compuesto por partículas, gases y vapores que se difunden rápida y fácilmente en el aire ambiente. Aunque los mecanismos de ventilación y separación pueden reducir las concentraciones de humo de tabaco, ni siquiera los sistemas comerciales más sofisticados pueden asegurar la total eliminación del humo. Por tanto, la utilización de sistemas de ingeniería para el control del HTA necesariamente implica la presencia de contaminación residual que, ante la falta de límites seguros de exposición, incrementaría innecesariamente el riesgo a la salud.

La utilización del recambio de aire y la inyección de 30 litros de aire por segundo por persona propuestas en las Fracciones I y III del Artículo 63 son recomendaciones técnicas para zonas de fumar que ya no se consideran válidas. Estas recomendaciones fueron generadas por la Asociación Norteamericana de Calefacción, Refrigeración y Aire Acondicionado (ASHRAE por sus siglas en inglés) en su estándar $62.1 .^{20}$ Sin embargo, en 2005, esta misma Asociación presentó un documento de posicionamiento respecto al HTA, donde establece que aunque la ventilación y separación de áreas son mecanismos que pueden reducir la exposición al HTA, “... la única forma de eliminar efectivamente los riesgos a la salud asociados con la exposición en interiores es prohibir el consumo de tabaco". ${ }^{11}$ Asimismo, especifica que ningún mecanismo de ventilación, filtración o purificación del aire es confiable para controlar los riesgos a la salud derivados de la exposición al HTA en los espacios donde se fuma. ${ }^{11}$ Finalmente la ASHRAE "....apoya la eliminación del consumo de tabaco en ambientes interiores como la manera óptima de minimizar la exposición al HTA". ${ }^{11}$ En acuerdo con este posicionamiento, la última versión del estándar 62.1 ya no proporciona recomendaciones de ventilación para espacios interiores donde se consume tabaco. ${ }^{21}$

La utilización de presión negativa en las zonas de fumar requerida en la Fracción IV del Artículo 63 ha sido descrita como uno de los métodos más eficaces para evitar el traslado de partículas a las áreas de no fumar. Sin embargo, los sistemas de presión negativa solo alcanzan una eficiencia del $80 \%$ asumiendo que la puerta de acceso no se abra más de 8 veces por hora (la eficacia disminuye al $50 \%$ si se abre 13 veces por hora). ${ }^{19}$ Así, aun bajo condiciones ideales y con el mejor sistema disponible, si una zona de fumar permite la entrada de 10 personas por hora, el humo de al menos dos personas se transmitirá a las áreas de no fumar.

\section{Conclusiones}

La evaluación de la LGCT y su Reglamento revela una clara insuficiencia para asegurar la protección de la 
población contra los efectos nocivos del HTA. Desde un punto de vista técnico no existen mecanismos capaces de evitar la transmisión de partículas o compuestos del HTA de las áreas interiores de fumar a las áreas de no fumar. La implementación de las áreas de fumar consideradas en el artículo 27 de la LGCT implica la contaminación de las áreas adyacentes donde está prohibido fumar, lo que produce un incremento en el riesgo a la salud derivado de la correcta aplicación de la legislación federal. Por ello, la LGCT debe ser reformada al eliminar la Fracción II del artículo 27 y reconocer a los espacios $100 \%$ libres de humo de tabaco como única estrategia eficaz para eliminar el HTA. Adicionalmente, la LGCT debe definir con claridad la distancia mínima de las áreas de fumar al aire libre a los espacios 100\% libres de humo de tabaco y eliminar la provisión de cualquier tipo de techo en las áreas para fumar al aire libre.

El fortalecimiento de la LGCT a través de la eliminación de áreas para fumar y la implementación a nivel federal de los espacios $100 \%$ libres de humo de tabaco, redundaría en una disminución del 20 al 30\% de la morbimortalidad asociada a la exposición al HTA en no fumadores. Los efectos positivos de esta medida no solo impactarían en la salud de los mexicanos, sino que también evitarían la pérdida de años productivos ocasionados por mortalidad y morbilidad temprana y reducirían los costos asociados al tratamiento de enfermedades asociadas al HTA. ${ }^{3}$

Los espacios $100 \%$ libres de humo de tabaco podrían beneficiar también la salud de los fumadores, pero dicho beneficio se anularía con la utilización de los mecanismos de control propuestos por la LGCT. Habitualmente se ha considerado expuestos al HTA únicamente a los no fumadores. Sin embargo, los fumadores están expuestos a concentraciones de HTA hasta cuatro veces más altas. ${ }^{22}$ Dado que el riesgo de cáncer de pulmón se incrementa conforme aumenta la exposición, un fumador expuesto a HTA tendrá un riesgo mayor de sufrir cáncer de pulmón que un fumador que no está expuesto. ${ }^{23}$ Dado que los sistemas de ventilación y separación propuestos en la LGCT son incapaces de eliminar el HTA del ambiente, su utilización resultaría en un incremento de la morbimortalidad en los fumadores, que no se observaría con la implementación de la prohibición completa al consumo de tabaco en áreas interiores. ${ }^{23}$ Incluso, la aplicación parcial de la LGCT podría resultar en un incremento substancial en la exposición de los fumadores al HTA. Por ejemplo, la separación de áreas sin incrementar la tasa de ventilación reduciría el volumen de aire disponible para la dilución del HTA, lo que incrementaría la concentración del contaminante en el área para fumar y los riesgos a la salud asociados a la exposición. Así, tanto para los fumadores como para los no fumadores, el desarrollo de espacios $100 \%$ libres de humo implica mayores beneficios a la salud que los que pueden obtenerse con sistemas mecánicos de control.

Además de los efectos positivos sobre la salud de fumadores y no fumadores, el desarrollo de espacios $100 \%$ libres de humo de tabaco tiene un efecto importante sobre la epidemia de tabaquismo activo. La implementación de una política claramente restrictiva al consumo de tabaco modifica la percepción social del tabaquismo y funciona como contrapeso a las medidas de promoción de las compañías tabacaleras. En países donde se ha aplicado una política estricta de prohibición al consumo de tabaco en áreas interiores se ha observado una disminución en la aceptación hacia el consumo de tabaco, reflejada en la disminución de la prevalencia de tabaquismo activo en adultos (4 a 10\%), la reducción del número de cigarros fumados por día (3.1 cigarros menos), la reducción en el inicio de consumo de tabaco entre adolescentes y el desarrollo de restricciones voluntarias al consumo en hogares y automóviles. ${ }^{2}$ Estos efectos positivos no se han observado en los países que han implementado medidas de control parcial, como las propuestas en la LGCT.

En conclusión, existe una clara necesidad de eliminar la exposición al HTA de todos los espacios públicos en México. Sin embargo, la LGCT recientemente aprobada es insuficiente para asegurar la protección a la salud de la población de los efectos nocivos del HTA. La existencia de áreas interiores para fumar representa un riesgo para la salud, ya que no existen mecanismos que eviten la transmisión de partículas a las áreas de no fumar. La prohibición completa del consumo de tabaco en áreas interiores redundaría en beneficios directos e indirectos a la salud de los mexicanos, tanto fumadores como no fumadores. Ante la evidencia científica que demuestra la imposibilidad de contar con mecanismos que eliminen la transmisión de partículas, la Fracción II del Artículo 27 debe ser derogada. Bajo el esquema actual, la correcta implementación del Artículo 27 no solo contravendría los objetivos planteados en el Artículo 5 de la LGCT, sino que también implicaría el desarrollo consciente y meditado de una situación de riesgo sanitario derivado de la legislación mexicana. Dado que la protección a la salud es un mandato constitucional, el Estado mexicano debe eliminar de la LGCT y su Reglamento cualquier disposición que implique el desarrollo o existencia de áreas para fumar en espacios interiores.

\section{Recomendaciones para la modificación de la LGCT y su Reglamento}

- Reconocimiento de los espacios $100 \%$ libres de humo de tabaco como única estrategia eficaz para 
la eliminación de los riesgos a la salud derivados de la exposición al HTA.

- Eliminación de la posibilidad de implementar áreas interiores para fumar, a través de la derogación de la Fracción II del Artículo 27 de la LGCT y los artículos correspondientes del Reglamento.

- Modificación de la definición de "espacio al aire libre" eliminando la posibilidad de que las áreas para fumar al aire libre tengan cualquier clase de estructura que funcione como techo (Art 2. Fracc. IV del RLGCT).

- Definición de las distancias mínimas de las áreas de fumar al aire libre a los accesos de los espacios 100\% libres de humo y a las zonas de paso obligatorio de los no fumadores.

- Ampliación de la definición de "espacio 100\% libre de humo de tabaco", al considerar que no sólo son áreas donde está prohibido fumar, sino donde también existen provisiones que aseguran la no contaminación desde espacios adyacentes.

\section{Declaración de conflicto de intereses}

Declaro no tener conflicto de intereses.

\section{Agradecimientos}

El presente ensayo es producto de investigaciones financiadas por el Consejo Nacional de Ciencia y Tecnología de México, con el proyecto "Exposición al Humo de Tabaco Ambiental, Salud y Productividad en Bares y Restaurantes de México" (S0008-2007-1-69760), del National Institute of Environmental Health Sciences a través del Center for Research in Environmental Diseases del MD Anderson Cancer Center (ES007784) y del Pilot Projects Program del National Institute of Occupational Safety and Health a través del Southwest Center for Occupational and Environmental Health de la Universidad de Texas School of Public Health (T42 $\mathrm{OH} 008421)$.

\section{Referencias}

I.World Health Organization.WHO Report on the Global Tobacco Epidemic, 2008:The MPOWER package. Geneva: 2008.

2. World Health Organization. Policy recommendations on protection from exposure to second-hand tobacco smoke. Geneva: 2007.

3.World Health Organization. WHO Framework Convention on Tobacco Control (WHO FCTC). Geneva: 2003.

4. Takala J. Introductory Report: Decent Work-Safe Work. 16th World

Congress on Safety and Health,Vienna, May. International Labor

Organization: 2002;27.

5. U.S. Department of Health and Human Services. The Health

Consequences of InvoluntaryExposure to Tobacco Smoke:A Report of the Surgeon General. U.S. Department of Health and Human Services. Atlanta, GA: 2006. [Consultado: 2010 jun 22]. Disponible en: http://www. surgeongeneral.gov/library/secondhandsmoke/

6. World Health Organization. International Consultation on Environmental Tobacco Smoke (ETS) and Child Health. Consultation Report. Geneva: 1999. [Consultado: 2010 jun 22]. Disponible en: http:// www.who.int/tobacco/research/en/ets_report.pdf

7. Drope J, Bialous SA, Glantz SA. Tobacco industry efforts to present ventilation as an alternative to smoke-free environments in North America. Tob Control 2004; Mar; 13 Suppl I:i4 I-7.

8. Scollo M, Lal A, Hyland A, Glantz S. Review of the quality of studies on the economic effects of smoke-free policies on the hospitality industry. Tob Control 2003; 12(I):13-20.

9. Griffith G,Welch C, Cardone A,Valdemoro A, Jo C. The global momentum for smokefree public places: best practice in current and forthcoming smokefree policies; [El momento global para espacios públicos libres de tabaco: mejores prácticas en políticas actuales y futuras]. Salud Publica Mex 2008;50(supl. 3):s299-308.

10. Cámara de Senadores de los Estados Unidos Mexicanos. Ley General para el Control del Tabaco 2008; Gaceta Oficial del Senado de la República 198:1/5-23. Fecha de publicación: 30/05/2008 II. Samet J, Bohanon Jr H, Coultas D, Houston T, Persily A, Schoen L, et al.ASHRAE position document on environmental tobacco smoke. American Society of Heating, Refrigerating and Air-Conditioning Engineers (ASHRAE).Atlanta, Georgia: 2005. [Consultado: 2010 jun 22]. Disponible en: http://www.ashrae.org/content/ASHRAE/ASHRAE/ArticleAltFormat/2 00582 I I239_347.pdf

12. Secretaría de Salud. Reglamento de la Ley General para el Control del Tabaco 2009; 3 I de Mayo de 2009; Diario Oficial de la Federación.

13. Mulcahy M, Evans DS, Hammond SK, Repace JL, Byrne M. Secondhand smoke exposure and risk following the Irish smoking ban: an assessment of salivary cotinine concentrations in hotel workers and air nicotine levels in bars. Tob Control 2005; Dec; 14(6):384-8.

14. State of Hawaii. Hawaii Smoke-Free Law 2006;Act 295. The Senate, Twenty-third legislature. [Consultado: 2010 jun 22]. Disponible en: http:// www.hawaiismokefree.com/the_law.html\#law

15. Illinois General Assembly. Smoke-Free Illinois Act 2008; I-I2008;SB0500 Public Act 095-00 17. Illinois General Assembly. [Consultado: 2010 jun 22]. Disponible en: http://www.lga.gov/legislation/publicacts/ fulltext.asp? Name $=095-1029$

16. Pion M, Givel M.Airport smoking rooms don't work. Tob Control 2004; I3:i37.

17. Invernizzi G, Ruprecht A, Mazza R, De Marco C, Boffi R. Transfer of particulate matter pollution from smoking to non-smoking coaches: the explanation for the smoking ban on Italian trains. Tob Control 2004;13(3):319.

18. Akbar-Khanzadeh F. Exposure to environmental tobacco smoke in restaurants without separate ventilation systems for smoking and nonsmoking dining areas. Arch Environ Health 2003; Feb;58(2):97-103. 19. Wagner J, Sullivan D, Faulkner D, Fisk W, Alevantis L, Dod R, et al. Environmental Tobacco Smoke Leakage from Smoking Rooms. Journal of Occupational and Environmental Hygiene 2004; I (2): I 10-8.

20.ASHRAE. Standard 62.I-200 I Ventilation for Acceptable Indoor Air Quality.American Society of Heating, Refrigerating and Air Conditioning Engineers. Atlanta, Georgia: 200I.

21.ASHRAE. Standard 62.I-2007Ventilation for Acceptable Indoor Air Quality.American Society of Heating, Refrigerating and Air Conditioning Engineers. Atlanta, Georgia: 2007.

22. Robinson J. Daily exposure to environmental tobacco smoke: smokers vs nonsmokers in California. Am J Public Health 1996;86(9): 1303-5. 23. Siegel M, Husten C, Merritt R, Giovino G, Eriksen M. Effects of separately ventilated smoking lounges on the health of smokers: Is this an appropriate public health policy.Tob Control 1995;4:22-9. 